\title{
Advances in Neuro-Oncology Imaging Techniques
}

\author{
Brannan E. O'Neill, BS, ${ }^{1}$ Christian B. Hochhalter, BA, ${ }^{1}$ Christopher Carr, BS, BA, ${ }^{1}$ Michael J. Strong, MD, PhD, MPH, \\ Marcus L. Ware, MD, PhD $^{2}$ \\ ${ }^{1}$ Tulane University School of Medicine, New Orleans, LA ${ }^{2}$ Department of Neurosurgery, Ochsner Clinic Foundation, New Orleans, LA
}

\begin{abstract}
Background: Central nervous system (CNS) tumors are a rare but devastating malignancy, often robbing patients of the basic quality of life. Despite advances in our understanding of the CNS tumor disease processes, the prognosis for patients with CNS tumors remains poor. Better characterization and diagnostic and monitoring approaches are necessary to assist in diagnosis and treatment of CNS tumors. One important tool in the neuro-oncology armamentarium is the use of advanced imaging techniques.
\end{abstract}

Methods: We searched PubMed using the keywords neuro-oncology imaging, pseudoprogression, molecular imaging, and biomarkers. We limited our search to full-text English articles and identified other relevant articles from the reference lists of previously identified articles.

Results: Advances in imaging techniques have allowed investigators to explore various imaging modalities, from tumor characterization to differentiating pseudoprogression from tumor progression. Better imaging can result in better diagnostic approaches, greater and safer resection techniques, and improved monitoring of tumor progression.

Conclusion: This review highlights advances in neuro-oncology imaging techniques and their clinical utility in the treatment and management of primary brain tumors.

Keywords: Biomarkers, glioma, magnetic resonance imaging, molecular imaging, multimodal imaging, surgical oncology

Address correspondence to Marcus L. Ware, MD, PhD, Department of Neurosurgery, Ochsner Clinic Foundation, 1514 Jefferson Hwy., New Orleans, LA 70121. Tel: (504) 842-4033. Email: mware@ochsner.org

\section{INTRODUCTION}

Primary brain tumors can arise from any cell type of the central nervous system (CNS). In the United States, the incidence of primary malignant and nonmalignant brain and other CNS tumors is 22.4 per 100,000 persons. ${ }^{1}$ Gliomas account for nearly $15 \%$ of all CNS neoplasms, yet these tumors constitute roughly $47 \%$ of all malignant brain tumors. ${ }^{1}$ The average annual age-adjusted mortality rate is disproportionately high at 4.32 per 100,000 persons, even though brain neoplasms make up less than $2 \%$ of all cancers. ${ }^{1}$ The 5 year survival rate for all malignant neoplasms of the brain is $34.9 \%$ across all demographics, according to the Central Brain Tumor Registry of the United States. ${ }^{1}$ Glioblastomas and anaplastic astrocytomas are the two most common histologic types of primary brain tumors and have poor 5-year survival rates of $5.5 \%$ and $29.7 \%$, respectively. ${ }^{1}$

Despite advances in our understanding of the disease mechanism and therapeutic options, overall survival rates remain dismal. However, favorable prognoses have been associated with factors including small preoperative tumor size, completeness of surgical resection, and lower pathologic grade. ${ }^{2}$ These factors are measurable, emphasizing the tangible need for better diagnostic approaches, greater and safer resection techniques, and improved monitoring of tumor progression. Neuroimaging plays a crucial role in all of these arenas, and this review highlights advances in neuro-oncology imaging techniques and their clinical utility in the treatment and management of primary brain tumors.

\section{NEURO-ONCOLOGY IMAGING TECHNIQUES}

The current standard of practice for radiographic evaluation of neuro-oncologic disease involves the use of computed tomography (CT) and magnetic resonance imaging (MRI). The advantages of CT imaging are convenience and speed. CT is less sensitive than MRI to motion artifact because the images are captured at a relatively rapid rate (Figure 1). High-density tissues such as bony structures or calcified tissues are easily differentiated on CT imaging. CT is also an excellent modality for identifying hemorrhage and can be used for a quick postoperative evaluation if hemorrhage into the tumor resection bed is a concern. Despite the advantages of CT imaging, identifying differences in soft tissues is challenging and requires the use of contrast agents to provide high-quality images, which is not always feasible in patients with renal disease or contrast allergies. ${ }^{3}$

MRI with gadolinium contrast is the gold standard for evaluating brain tumors pretherapy and posttherapy. For many neuro-oncology applications, MRI is superior to CT imaging because MRI provides better anatomic resolution without the use of ionizing radiation or iodine contrast medium. 


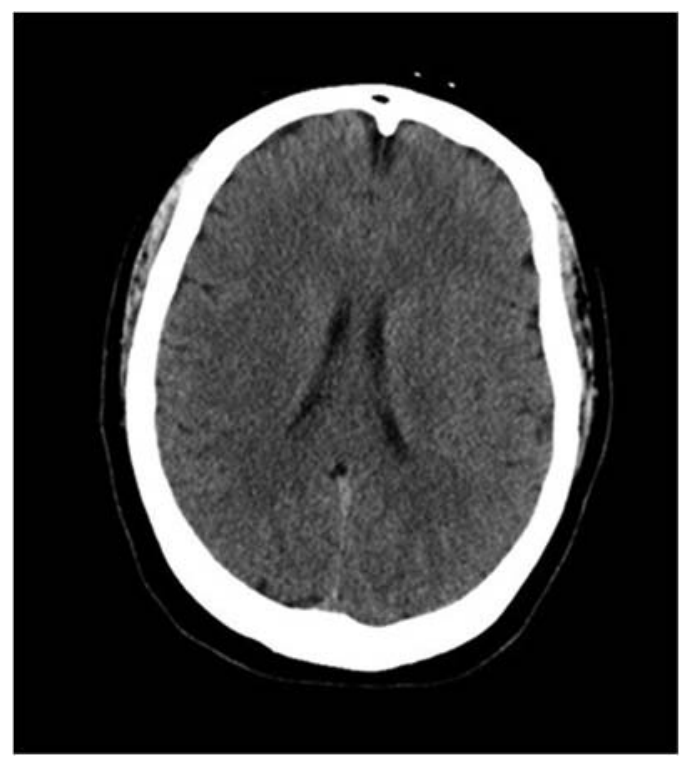

Figure 1. Computed tomography image of the brain.

Contrast-enhanced T1-weighted imaging highlights areas of blood-brain barrier breakdown but must be compared with non-contrast-enhanced T1 imaging to differentiate areas of interest from blood products, fat, or proteinaceous material (Figure 2). T2-weighted imaging is useful for discriminating nuances of tumor-related edema as this sequence is more sensitive than other MRI sequences to fluctuations in the brain's water content (Figure 3A). Fluid-attenuated inversion recovery (FLAIR) imaging suppresses cerebrospinal fluid (CSF) and delineates lesions adjacent to CSF-rich regions (Figure 3B). ${ }^{4}$ The sequence of a gradient-recalled echo (GRE) T2-weighted image has more accurate detection rates of small lesions compared to other MRI sequences because of its ability to pick up subtle changes in the magnetic field uniformity (Figure 3C). ${ }^{5}$

Advances in MRI software have expanded our ability to use this technology beyond the conventional T1 and T2 MRI. For example, diffusion-weighted imaging (DWI) sequences use the assumed motion patterns of water particles to map tissue architecture on a microscopic level (Figure 4A). Diffusion tensor imaging (DTI) provides information about the directional movements of tissue and can provide insight on spatial relationships of abnormal-or oncologic-borders compared to healthy white matter (Figure 4B) ${ }^{4,6}$ In diffusion imaging, a scalar value called the fractional anisotropy (FA) provides information about white matter myelination, fiber density, and axon diameter. Mean diffusivity (MD) and the apparent diffusion coefficient (ADC) can be used in combination with the FA to measure the integrity of neuronal tissue and mark fiber loss. ${ }^{7}$ Dynamic imaging plays a significant role in neurooncologic imaging, with perfusion MRI demonstrating the vascularity associated with intracranial tumors. Contrast agent flows through the cerebrovascular system in dynamic susceptibility contrast (DSC) MRI, but the permeability of neoplasms can also be assessed using dynamic contrastenhanced (DCE) magnetic resonance perfusion imaging. ${ }^{8}$ For noninvasive imaging of metabolic profiles of brain tumors and histologic classification, magnetic resonance spectroscopy (MRS) is a popular modality because of its ability to measure concentrations of molecules in brain tissue. ${ }^{9,10}$

Nuclear imaging techniques such as positron emission tomography (PET) and single-photon emission computed tomography (SPECT) provide functional brain imaging, with SPECT tailored to detecting metabolic processes (Figure $5 A) .{ }^{11}$ PET technology takes advantage of short-lived positron emitters, usually $11 \mathrm{C}$ and $18 \mathrm{~F}$ (Figure $5 \mathrm{~B}$ ). These radioisotopes produce gamma rays that are detected in the PET scanner to produce an image. In addition, the radioisotopes are linked to a tracer molecule that can be taken up by cells or bind to specific receptors (eg, neuroreceptors). The ability to assess both the spatial and temporal activity of neuroreceptors and thereby assess brain function makes PET imaging an important modality in clinical neuroimaging. While PET imaging is ideal for assessing brain function, its ability to differentiate structural aberrations in the CNS is limited. ${ }^{3}$ Further, the use of radioactive glucose in cancer surveillance has limited sensitivity in the brain because of the high baseline metabolic rate. Finally, because PET imaging uses x-rays, the radiation risk involved in using this modality is a concern.

\section{DIFFERENTIATING TUMOR PROGRESSION, PSEUDOPROGRESSION, AND RADIATION NECROSIS}

Pseudoprogression is a posttreatment phenomenon that appears similar on imaging to true tumor progression.
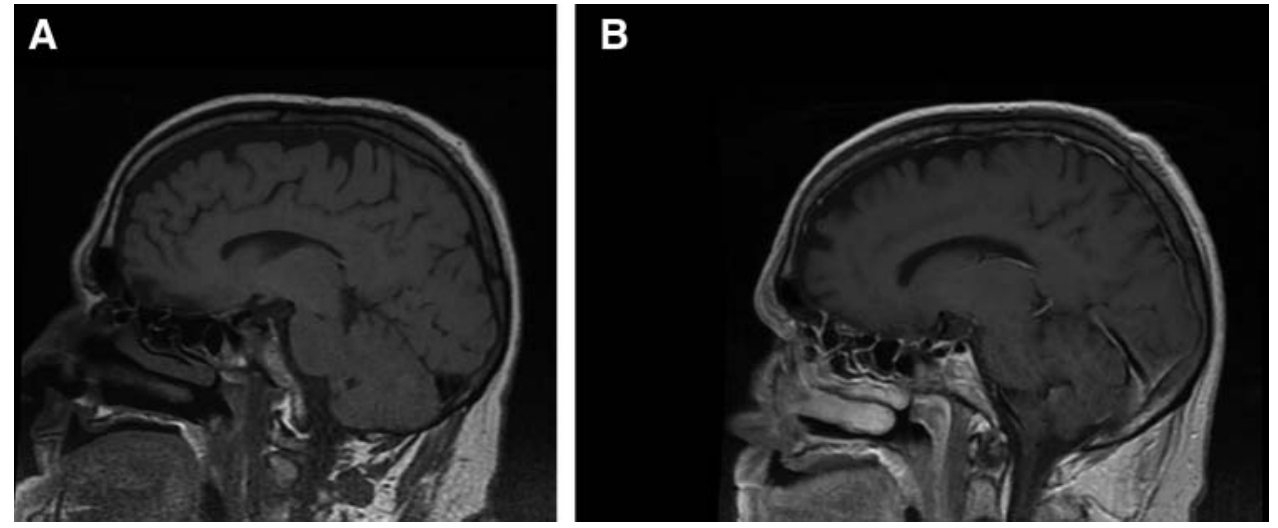

Figure 2. T1-weighted magnetic resonance image of the brain (A) without contrast and (B) with contrast. 

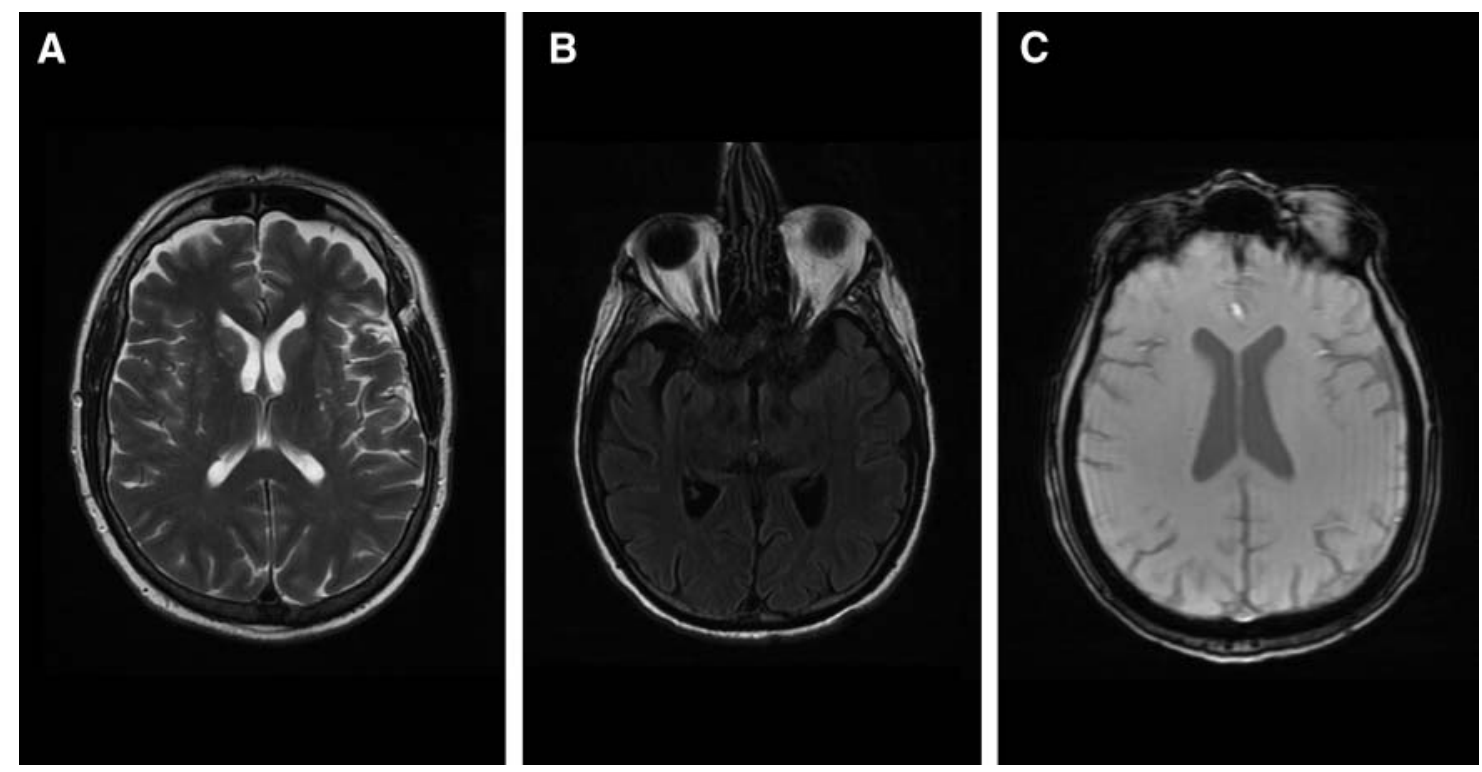

Figure 3. (A) T2-weighted magnetic resonance image of the brain; (B) fluid attenuated inversion recovery (FLAIR) image of the brain; (C) T2-weighted gradient-recalled echo (GRE) image of the brain.

Pseudoprogression occurs because of localized edema and subsequent contrast enhancement, but it is a benign condition that usually regresses. Distinguishing pseudoprogression from true tumor progression is often difficult, but differentiating between the two conditions drastically changes management. ${ }^{12}$ Distinguishing from radiation necrosis is also warranted, as radiation necrosis is an unwanted but known sequela of treatment and demands different management. Imaging modalities that can be used to monitor the metabolic properties of tumors are being investigated and include CT perfusion, magnetic resonance perfusion, DWI, MRS, PET, and SPECT. ${ }^{13-15}$ Although each modality is important in assessing CNS disease pathology, they have limitations. For example, MRI does not adequately differentiate tumor recurrence from the effects of radiation. ${ }^{13}$ Other mo- dalities, such as MRS and PET, may lead to false-positive determinations of tumor recurrence. ${ }^{16}$

Because of the ambiguous nature of identifying true tumor progression from pseudoprogression on imaging and the importance of differentiating between the two, intense research is currently underway. Injury caused by radiation and true tumor recurrence can be differentiated with a combination of DWI and MRS with ADC ratio, as the ADC has been shown to be increased in necrotic tissue compared to recurrent tumor tissue. ${ }^{17} \mathrm{~A}$ metaanalysis published in 2015 showed that SPECT had good diagnostic ability to differentiate tumor recurrence from radiation necrosis. ${ }^{18} \mathrm{~A}$ 2015 study showed that 18F-fluoro-ethyl-tyrosine (18F-FET) PET differentiated tumor progression from treatment-related nonneoplastic changes with greater accuracy than
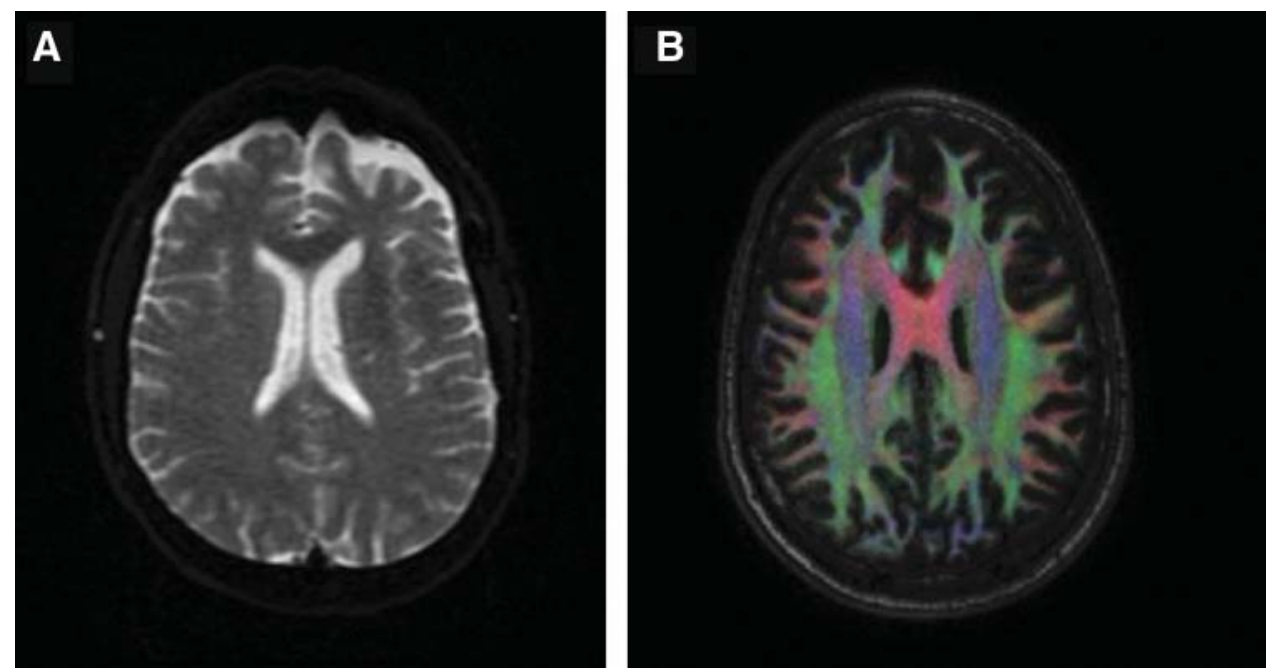

Figure 4. (A) Diffusion-weighted image (DWI) of the brain; (B) diffusion tensor image (DTI) of the brain. 

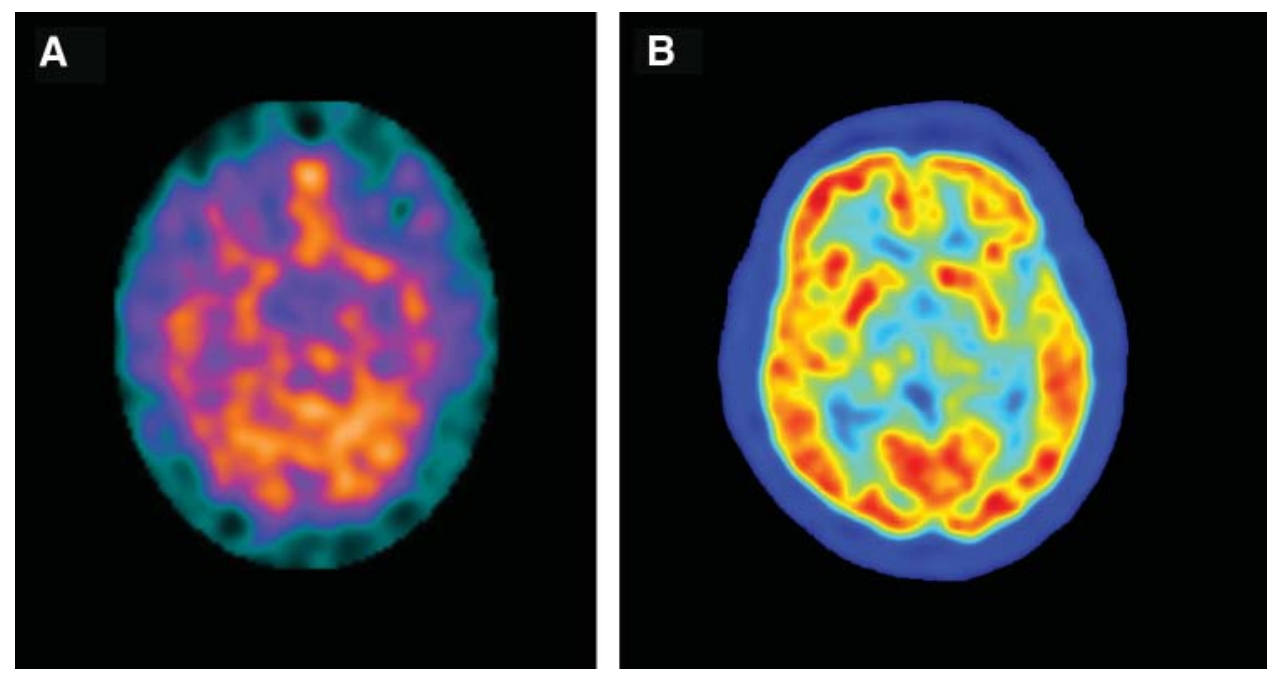

Figure 5. (A) Single photon emission computed tomography (SPECT) image of the brain. (Reprinted from Vigren P et al. ${ }^{11}$ Copyright 2014 licensed by Creative Commons Attribution License [CC BY].) (B) Positron emission tomography (PET) image of the brain. (Image credited to Jens Maus, Wikimedia Commons [http://en.wikipedia.org/wiki/Image:PET-image.jpg] and released into the public domain.)

conventional MRI protocols. ${ }^{19}$ Several studies have suggested that DSC and DCE perfusion imaging can reliably distinguish between posttreatment findings and true tumor progression, but further standardized investigation is warranted before these techniques are used in the clinic. ${ }^{20}$

\section{NONINVASIVE CLASSIFICATION OF ADULT BRAIN TUMORS}

Advances in imaging techniques can augment traditional image modalities (eg, CT and MRI) by providing improved characterization of brain lesions. For example, by using a radiotracer that is taken up preferentially by glioma cells, 18FFET PET delivers superior demarcation of tumor margins grossly. ${ }^{21}$ Further, by measuring the activity of 18F-FET uptake over time, distinguishing between low-grade and highgrade gliomas is feasible. The most significant prognostic factor related to tumor grade is the minimum time to peak uptake (TTPmin). ${ }^{22}$ This variable allows for reliable prediction of prognosis, with an early TTPmin correlating to graver outcomes than tumors with a late TTPmin. By analyzing this factor, glioma patients can be stratified by risk and predicted tumor progression, potentially influencing patient management.

One area of active investigation that has shown promise in the field of neuro-oncology is the use of magnetic nanoparticles (MNPs) for diagnostic and therapeutic purposes. MNPs can be modified to carry peptides or antibodies directed against cancer cell targets. Because of their magnetic properties, these particles augment standard imaging protocols. MNPs accumulate in CNS lesions and appear as hypointense regions on T2-weighted GRE. The most effective type of MNP for imaging is the ultrasmall superparamagnetic iron oxide nanoparticle (USPIONP). This particle has a greater half-life than the standard MNP and thus can be viewed on $\mathrm{MRI}$ for a longer time period. ${ }^{23}$ USPIONPs are useful for tracking tumor recurrence as they detect areas of heightened vascularity and regions of pseudoprogression posttreatment. $^{24}$
Defining features seen on MRI can distinguish between phenotypes of glioblastoma multiforme (GBM) because of their distinct molecular pathways. Using gadoliniumenhanced MRI, 3 distinct clusters of phenotypic features can provide prognostic indications: pre-multifocal, spherical, and rim-enhancing. These subsets of identifiable imaging features can be mapped to molecular signaling pathways to yield GBM classification, risk stratification, and individualized treatment approaches. ${ }^{25}$

\section{TUMOR MOLECULAR BIOMARKERS}

Physiologic markers of tumors, specifically molecular and genetic variants, can aid advanced structural imaging in identifying, treating, and monitoring brain tumors. Isocitrate dehydrogenase 1 and 2 (IDH1 and IDH2) genes can be disrupted in primary glioblastomas, secondary glioblastomas, and oligodendrogliomas. Tumors with IDH1 aberrations appear as multifocal invasive masses with little to no detectable contrast enhancement. The IDH1 and IDH2 enzymes both have the capacity to convert alpha-ketoglutarate into 2hydroxyglutarate $(2 \mathrm{HG})$ that can be detected by proton MRS (2HG-MRS). ${ }^{26}$ Integrating 2HG-MRS with standard $\mathrm{MRI}$ protocols enables the ability to monitor the abundance of IDH-mutant tumor cells noninvasively and may be used to monitor disease recurrence and therapeutic response. ${ }^{27} \mathrm{Co}-$ deletion of chromosomes $1 p$ and $19 q$ is detected in approximately $80 \%$ of oligodendrogliomas, and tumors with this characteristic demonstrate irregular margins. ${ }^{28}$ The T1 and T2 signal features are more heterogeneous than in tumors without $1 \mathrm{p} / 19 \mathrm{q}$ codeletions. ${ }^{29}$ Mutated versions of the epidermal growth factor receptor (EGFR) lead to enhanced tumorigenesis in glioblastomas. Using MRI to evaluate tumors with EGFR amplification shows increased ratios of contrastenhancing tissue compared to necrotic tissue, increased ratios of T2 to contrast-enhancing volume, decreased acuity of T2 borders, and lower ADC values. ${ }^{30,31}$ Tumors with EGFR amplification, phosphatase and tensin homolog (PTEN) 
deletion, and unmethylated O6-methylguanine-DNA methyltransferase (MGMT) show increased tumor blood volume. ${ }^{32}$

The $18 \mathrm{kDa}$ translocator protein (TSPO) is a mitochondrial protein that is predominately expressed in neoplastic cells, including tumors involving the CNS. ${ }^{33}$ Taking advantage of this specificity in expression and the potential to serve as a target for imaging, Tsartsalis and colleagues evaluated the feasibility and clinical utility of using a TSPO-specific radioiodinated tracer (CLINDE) in conjunction with SPECT imaging. ${ }^{34}$ Although the study was conducted in a mouse glioma model, the results demonstrated the potential for clinical application in neuroimaging.

\section{CONCLUSION}

Advances in imaging of the brain have allowed for effective, noninvasive approaches to diagnosing and treating CNS tumors. Further research and explorative studies are needed to distinguish unsolved nuances of brain tumors and treatment-related findings. Advances in neuroimaging offer the opportunity to enhance the management and care of patients with brain tumors, thereby improving their overall survival.

\section{ACKNOWLEDGMENTS}

This work was supported by a Ruth L. Kirschstein National Research Service Award F30CA177267 from the National Cancer Institute to Dr Michael Strong. Otherwise, the authors have no financial or proprietary interest in the subject matter of this article.

\section{REFERENCES}

1. Ostrom QT, Gittleman $\mathrm{H}, \mathrm{Xu}$ J, et al. CBTRUS Statistical Report: Primary Brain and Other Central Nervous System Tumors Diagnosed in the United States in 2009-2013. Neuro Oncol. 2016 Oct;18(suppl_5):v1-v75. doi: 10.1093/neuonc/now207.

2. Wen PY, Fine HA, Black PM, Shrieve DC, Alexander E 3rd, Loeffler JS. High-grade astrocytomas. Neurol Clin. 1995 Nov;13 (4):875-900.

3. Mier W, Mier D. Advantages in functional imaging of the brain. Front Hum Neurosci. 2015 May 19;9:249. doi: 10.3389/ fnhum.2015.00249.

4. Cha S. Neuroimaging in neuro-oncology. Neurotherapeutics. 2009 Jul;6(3):465-477. doi: 10.1016/j.nurt.2009.05.002.

5. Tang MY, Chen TW, Zhang XM, Huang XH. GRE T2*-weighted MRI: principles and clinical applications. Biomed Res Int. 2014;2014:312142. doi: 10.1155/2014/312142.

6. Lequin M, Hendrikse J. Advanced MR imaging in pediatric brain tumors, clinical applications. Neuroimaging Clin N Am. 2017 Feb;27(1):167-190. doi: 10.1016/j.nic.2016.08.007.

7. Risacher SL, Saykin AJ. Neuroimaging biomarkers of neurodegenerative diseases and dementia. Semin Neurol. 2013 Sep;33(4):386-416. doi: 10.1055/s-0033-1359312.

8. Essig M, Shiroishi MS, Nguyen TB, et al. Perfusion MRI: the five most frequently asked technical questions. AJR Am J Roentgenol. 2013 Jan;200(1):24-34. doi: 10.2214/AJR.12.9543.

9. Siasios I, Valotassiou V, Kapsalaki E, et al. Magnetic resonance spectroscopy and single-photon emission computed tomography in the evaluation of cerebral tumors: a case report. J Clin Med Res. 2017 Jan;9(1):74-78.

10. van der Graaf M. In vivo magnetic resonance spectroscopy: basic methodology and clinical applications. Eur Biophys $J$. 2010 Mar;39(4):527-540. doi: 10.1007/s00249-009-0517-y.
11. Vigren $P$, Engström $M$, Landtblom AM. SPECT in the KleineLevin syndrome, a possible diagnostic and prognostic aid? Front Neurol. 2014 Sep 23;5:178. doi: 10.3389/fneur.2014.00178.

12. Brandsma D, Stalpers L, Taal W, Sminia P, van den Bent MJ. Clinical features, mechanisms, and management of pseudoprogression in malignant gliomas. Lancet Oncol. 2008 May;9(5):453-461. doi: 10.1016/S1470-2045(08)70125-6.

13. Chuang MT, Liu YS, Tsai YS, Chen YC, Wang CK. Differentiating radiation-induced necrosis from recurrent brain tumor using MR perfusion and spectroscopy: a meta-analysis. PLoS One. 2016 Jan 7;11(1):e0141438. doi: 10.1371/journal.pone.0141438.

14. Verma N, Cowperthwaite MC, Burnett MG, Markey MK. Differentiating tumor recurrence from treatment necrosis: a review of neuro-oncologic imaging strategies. Neuro Oncol. 2013 May;15(5):515-534. doi: 10.1093/neuonc/nos307.

15. Wan B, Wang S, Tu M, Wu B, Han P, Xu H. The diagnostic performance of perfusion MRI for differentiating glioma recurrence from pseudoprogression: a meta-analysis. Medicine (Baltimore). 2017 Mar;96(11):e6333. doi: 10.1097/ MD.0000000000006333.

16. Mitsuya K, Nakasu Y, Horiguchi S, et al. Perfusion weighted magnetic resonance imaging to distinguish the recurrence of metastatic brain tumors from radiation necrosis after stereotactic radiosurgery. J Neurooncol. 2010 Aug;99(1):81-88. doi: 10.1007/s11060-009-0106-z.

17. Zeng QS, Li CF, Liu H, Zhen JH, Feng DC. Distinction between recurrent glioma and radiation injury using magnetic resonance spectroscopy in combination with diffusion-weighted imaging. Int J Radiat Oncol Biol Phys. 2007 May 1;68(1):151-158.

18. Zhang $\mathrm{H}, \mathrm{Ma} \mathrm{L}, \mathrm{Wu} C, \mathrm{Xu} B N$. Performance of SPECT in the differential diagnosis of glioma recurrence from radiation necrosis. J Clin Neurosci. 2015 Feb;22(2):229-237. doi: 10.1016/j. jocn.2014.06.102.

19. Galldiks N, Stoffels G, Filss C, et al. The use of dynamic O-(218F-fluoroethyl)-I-tyrosine PET in the diagnosis of patients with progressive and recurrent glioma. Neuro Oncol. 2015 Sep;17 (9):1293-1300. doi: 10.1093/neuonc/nov088.

20. Patel $P$, Baradaran $H$, Delgado $D$, et al. MR perfusion-weighted imaging in the evaluation of high-grade gliomas after treatment: a systematic review and meta-analysis. Neuro Oncol. 2017 Jan;19(1):118-127. doi: 10.1093/neuonc/now148.

21. Westphal M, Lamszus K. Circulating biomarkers for gliomas. Nat Rev Neurol. 2015 Oct;11(10):556-566. doi: 10.1038/ nrneurol.2015.171.

22. Jansen NL, Suchorska B, Wenter V, et al. Prognostic significance of dynamic 18F-FET PET in newly diagnosed astrocytic highgrade glioma. J Nucl Med. 2015 Jan;56(1):9-15. doi: 10.2967/ jnumed.114.144675.

23. Mahmoudi K, Hadjipanayis CG. The application of magnetic nanoparticles for the treatment of brain tumors. Front Chem. 2014 Dec 3;2:109. doi: 10.3389/fchem.2014.00109.

24. Gambarota G, Leenders W, Maass C, et al. Characterisation of tumour vasculature in mouse brain by USPIO contrastenhanced MRI. Br J Cancer. 2008 Jun 3;98(11):1784-1789. doi: 10.1038/sj.bjc.6604389.

25. Itakura H, Achrol AS, Mitchell LA, et al. Magnetic resonance image features identify glioblastoma phenotypic subtypes with distinct molecular pathway activities. Sci Transl Med. 2015 Sep 2;7(303):303ra138. doi: 10.1126/scitransImed.aaa7582.

26. Andronesi OC, Kim GS, Gerstner E, et al. Detection of 2hydroxyglutarate in IDH-mutated glioma patients by in vivo spectral-editing and $2 \mathrm{D}$ correlation magnetic resonance spectroscopy. Sci Transl Med. 2012 Jan 11;4(116):116ra4. doi: 10.1126/scitranslmed.3002693. 
27. de la Fuente MI, Young RJ, Rubel J, et al. Integration of 2hydroxyglutarate-proton magnetic resonance spectroscopy into clinical practice for disease monitoring in isocitrate dehydrogenase-mutant glioma. Neuro Oncol. 2016 Feb;18 (2):283-290. doi: 10.1093/neuonc/nov307.

28. Kraus JA, Koopmann J, Kaskel P, et al. Shared allelic losses on chromosomes $1 p$ and $19 q$ suggest a common origin of oligodendroglioma and oligoastrocytoma. J Neuropathol Exp Neurol. 1995 Jan;54(1):91-95.

29. Jenkinson MD, du Plessis DG, Smith TS, Joyce KA, Warnke PC, Walker C. Histological growth patterns and genotype in oligodendroglial tumours: correlation with MRI features. Brain. 2006 Jul;129(Pt 7):1884-1891.

30. Young RJ, Gupta A, Shah AD, et al. Potential role of preoperative conventional MRI including diffusion measurements in assessing epidermal growth factor receptor gene amplification status in patients with glioblastoma. AJNR Am J Neuroradiol. 2013 Dec;34(12):2271-2277. doi: 10.3174/ajnr. A3604.
31. Diehn M, Nardini C, Wang DS, et al. Identification of noninvasive imaging surrogates for brain tumor geneexpression modules. Proc Natl Acad Sci U S A. 2008 Apr 1;105 (13):5213-5218. doi: 10.1073/pnas.0801279105.

32. Ryoo I, Choi SH, Kim JH, et al. Cerebral blood volume calculated by dynamic susceptibility contrast-enhanced perfusion MR imaging: preliminary correlation study with glioblastoma genetic profiles. PLoS One. 2013 Aug 19;8(8):e71704. doi: 10.1371/journal.pone.0071704.

33. Cosenza-Nashat M, Zhao ML, Suh HS, et al. Expression of the translocator protein of $18 \mathrm{kDa}$ by microglia, macrophages and astrocytes based on immunohistochemical localization in abnormal human brain. Neuropathol Appl Neurobiol. 2009 Jun;35(3):306-328. doi:10.1111/j.1365-2990.2008.01006.x.

34. Tsartsalis S, Dumas N, Tournier BB, et al. SPECT imaging of glioma with radioiodinated CLINDE: evidence from a mouse GL26 glioma model. EJNMMI Res. 2015 Mar 13;5:9. doi: 10.1186/ s13550-015-0092-4.

This article meets the Accreditation Council for Graduate Medical Education and the American Board of Medical Specialties Maintenance of Certification competencies for Patient Care, Medical Knowledge, and Practice-Based Learning and Improvement. 